\title{
ON CONVERGENCE OF THE FINITE-ELEMENT METHOD FOR A CLASS OF ELASTIC-PLASTIC SOLIDS*
}

\author{
BY \\ K. S. HAVNER AND H. P. PATEL** \\ North Carolina State University, Raleigh
}

\begin{abstract}
A proof of convergence of the finite-element method in rate-type, quasistatic boundary value problems is presented. The bodies considered may be discretely heterogeneous and elastically anisotropic, their plastic behavior governed by historydependent, piecewise-linear yield functions and fully coupled hardening rules. Elastic moduli are required to be positive-definite and plastic moduli nonnegative-definite. Precise and complete arguments are given in the case of bodies whose surfaces are piecewise plane.
\end{abstract}

1. Introduction. In [1] the senior author presented a convergence argument for a discretized aggregate model in polycrystalline plasticity. In essence the argument constituted a proof of convergence of the finite element method within that context. Here we substantially modify and extend the previous proof to a broader class of boundary-value problems in theoretical plasticity. Our concern is bodies which may be discretely heterogeneous and whose constituents are characterized by anisotropic elastic properties and piecewise-linear, history-dependent yield functions with fully coupled hardening rules.

We address the following question: given the current distribution of stress and constitutive properties within an elastic-plastic solid, does the instantaneous response of a suitablychosen finite-element model converge to the true instantaneous response as the model is refined? We prove that the answer to this question is yes. The deeper and more difficult problem of establishing absolute convergence of the finite-element method for a sequence of incremental solutions is not undertaken. Although the latter is the real issue in actual calculations, convergence of the rate-type problem in our sense is certainly a necessary test for the finite element method and of itself is not trivial.

The proof in [1] was motivated by Tong and Pian's convergence arguments in lirear elasticity [2] and was patterned in part after their approach. Here, in addition to broadening the class of problems considered, we have found a more straightforward proof of convergence than in [1]. In achieving this new proof we have greatly benefitted from the clear and engaging analysis by Strang and Fix [3].

2. Constitutive equations. Consider a history-dependent elastic domain bounded by a finite set of hyperplanes of fixed orientation in (Cauchy) stress space. Let $d_{k}$ denote

${ }^{*}$ Received March 16, 1974; revised version received September 24, 1974. This work was supported by the National Science Foundation, Solid Mechanics Program, through Grant GK-31313.

** Present address: Firestone Tire \& Rubber Co., Akron, Ohio. 
the current algebraic distance from the origin to the $k$ th hyperplane and $N_{k}$ denote the unit outward normal. Then $d_{k}$ is positive or negative as the scalar product of $N_{k}$ with the position vector to any point in this plane is positive or negative (i.e., the elastic domain need not contain the origin). An admissible stress state must satisfy $N_{k} \sigma \leq d_{k}$ for all $k$. (Juxtaposition implies the scalar inner product.) A critical state of stress is defined by the condition

$$
\Phi_{k}(\sigma) \equiv N_{k} \sigma-d_{k}=0
$$

for at least one $k$.

The general theory of associated flow-laws asserts that the $\Phi_{k}$ are potentials for plastic strain-rate $\epsilon^{p}$, whence

$$
\epsilon^{p}=\gamma_{k}\left(\partial \Phi_{k} / \partial \sigma\right)=\gamma_{k} N_{k} .
$$

The nonnegative plastic multipliers $\gamma_{k}$ are scalar rates, with summation in (2) over all hyperplanes passing through the (critical) stress point $\sigma$. Further, in an increment of straining $\dot{\Phi}_{k} \leq 0$ for each of these critical hyperplanes. Thus, if we denote stress rate by $\tau$,

$$
N_{k} \tau-\dot{d}_{k} \leq 0
$$

for every $k$ satisfying (1). (Alternatively, all "rates" may be understood as incremental changes.)

The changing shape and position of the elastic domain are defined by relating the rates of change of all the distances $d_{k}$ to the scalar rates $\gamma_{k}$ through a general hardening rule

$$
\dot{d}_{k}=h_{k i} \gamma_{i}
$$

so that

$$
d_{k}=\int h_{k i} \gamma_{i} d \theta+d_{k}^{\circ},
$$

where $\theta$ is a time-like variable and the integration is taken over the history of the deformation. (The $d_{k}{ }^{\circ}$ define the elastic domain in the virgin, unstressed state.) The $h_{k i}$ may be functionals of the deformation history but are taken to be independent of the rates $\gamma_{i}$, and their instantaneous (current) values are supposed known. Furthermore, either $\gamma_{k}=0$ or the equality in (3) is satisfied.

The inequalities and hardening rule may be summarized as

$$
\begin{array}{r}
h_{k} \gamma_{i} \geq N_{k} \tau, \quad \gamma_{k} \geq 0, \\
\gamma_{k} h_{k i} \gamma_{i}=\gamma_{k} N_{k} \tau \quad(\text { for each } k) .
\end{array}
$$

Lastly, the total (Eulerian) strain-rate $\epsilon$ is given by

$$
\epsilon \equiv \partial u=\mathscr{L}^{-1} \tau+\gamma_{k} N_{k},
$$

where $\partial u$ denotes the symmetric gradient of displacement rate $u$ and $\mathfrak{L}$ is the tensor (or matrix) of elastic moduli, considered to be symmetric positive-definite herein. 
In the context of crystalline slip models, where $\sqrt{ } 2 \gamma_{k}$ is the plastic shear rate in the $k$ th crystallographic slip system and $d_{k} / \sqrt{ } 2$ is the corresponding critical shear strength, a hardening rule of the general type (4) was first proposed by Taylor [4, 5]: $h_{k i}=2 H$ for all $k, j$ ( $H$ denoting the single slip modulus). Taylor's rule has had extensive application in crystalline aggregate calculations by Bishop and Hill [6], Iin (see [7] for a complete bibliography), Hutchinson [8, 9], and Havner, et al. [10, 11]. Budiansky and $\mathrm{Wu}$ [12] adopted a rule which may be expressed $h_{k j}=H N_{k} N_{j}$, and Koiter [13], without regard to a particular context, suggested the uncoupled rule $h_{k i}=2 H \delta_{k i}\left(\delta_{k i}\right.$ the Kronecker delta). The general hardening rule may be attributed to Mandel [14] and Hill [15].

The equations above are not necessarily limited to crystal plasticity, however. Constitutive relations of the form (6)-(8) also have been adopted for the analysis of elastic-plastic structures. In that context Maier has presented a similar but broader theory which incorporates the possibility of nonassociated flow laws and "work-softening" moduli $h_{k i}$ (cf. $\left.[16,17]\right)$. Even in the case of elastically isotropic materials, where one may wish to consider the von Mises criterion, say, as a norm, an approximating, piecewise linear yield surface can always be defined (see [18], for example). Then Taylor's rule corresponds to classical isotropic hardening (i.e. an expanding elastic domain, with all $d_{k}$ equal), whereas $h_{k i}=H N_{k} N_{i}, H$ constant, represents Reuss-Prager "kinematic" hardening [19].

(Implicit in the above is the assumption that additional deformations and rotations are sufficiently small so that no distinction need be made between nominal, co-rotational, and convected stress rates. In applications to metal plasticity this is adequate so long as $\epsilon^{p}$ is no more than an order of magnitude greater than $\mathscr{L}^{-1} \tau$ for ordinary values of the moduli.)

3. A minimum principle. Henceforth we require the $h_{k i}$ to be symmetric, nonnegative-definite. Although a broader theory (as in $[16,17]$ ) may be physically plausible, this specification is general enough to include the various particular cases actually adopted in calculations. Moreover, it makes possible the establishment of certain minimum principles which are the foundation of our convergence proof herein.

Consider an elastic-plastic solid occupying a volume $V$ in Euclidean space and let $x$ denote the position vector of material point $X$ with respect to an appropriate reference frame. We suppose the current values of $\sigma, d_{k}$, and $h_{k i}$ are known throughout $V$ and that $\sigma(x)$ is an equilibrium stress field (restricting consideration to quasi-static processes). The elastic moduli $\mathfrak{L}$ are assumed to be spatially uniform locally and independent of the deformation history but, of course, may change across material interfaces in a heterogeneous solid. In the rate-type boundary value problem that is our concern, body force rates $f$ are prescribed throughout $V$, traction rates $t$ are prescribed on a portion of the surface $A_{F}$, and displacement rates $u$ are prescribed on the remainder of the surface $A_{D}$.

We now introduce the scalar functional for which an overall minimum principle can be proved (patterning our notation after that in Strang and Fix [3]). Define

$$
I(v)=\frac{1}{2} a(v, v)-\frac{1}{2}(\lambda, v)-(f, v)
$$

over the space $\mathrm{JC}^{1}$ of continuous (vector) functions $v(x)$ satisfying $v=u$ on $A_{D}$ (i.e., the space of kinematically admissible displacement rates), where 


$$
\begin{aligned}
a(v, v) & =\int \partial v(x) \mathfrak{L} \partial v(x) d V, \\
(\lambda, v) & =\int \partial v(x) \mathfrak{L} N_{k} \lambda_{k}(x) d V, \\
(f, v) & =\int v(x) f d V+\int v(x) t d A_{k},
\end{aligned}
$$

and the $\lambda_{k}$ are related to $v$ through (6)-(8):

$$
\begin{gathered}
h_{k j} \lambda_{i} \geq N_{k} \zeta, \quad \lambda_{k} \geq 0, \\
\lambda_{k} h_{k j} \lambda_{i}=\lambda_{k} N_{k} \zeta \quad(\text { for each } k), \\
\zeta=\mathcal{L}\left(\partial v-N_{k} \lambda_{k}\right) .
\end{gathered}
$$

(The positive-definite quadratic form $a(v, v)$ is conveniently called the energy in the function $v(x)$.) The following theorem then may be stated: the statically admissible true solution $u(x)$ minimizes I on the admissible class $v(x)$. The proof is as follows.

From virtual work (equivalently, the Gauss-Green transformation)

$$
\int \tau(u) \partial v d V=(f, v)+\int u t(u) d A_{D},
$$

where $\tau(u)$ is the true stress-rate. Thus, since $a(v, v)-(\lambda, v)=\int \zeta(v) \partial v d V$,

$$
\begin{aligned}
& I(v)=\frac{1}{2} \int(\zeta-2 \tau) \partial v d V+\int u t(u) d A_{D} . \\
& I(u)=-\frac{1}{2} \int \tau \partial u d V+\int u t(u) d A_{D} .
\end{aligned}
$$

Hence, with the aid of (6)-(8) and (11),

$$
\begin{aligned}
& I(v)-I(u)=\frac{1}{2} \int\left\{(\tau-\zeta) \mathfrak{L}^{-1}(\tau-\zeta)\right. \\
& \left.\quad+\left(\gamma_{k}-\lambda_{k}\right) h_{k i}\left(\gamma_{i}-\lambda_{i}\right)+2 \lambda_{k}\left(h_{k i} \gamma_{i}-N_{k} \tau\right)\right\} d V \geq 0,
\end{aligned}
$$

which completes the proof. (This result also was obtained in [1]. The proof of a minimum principle for invertible $h_{k i}$ was given by Hill [15]. For a continuously smooth yield function and the associated flow rule of Prager [19] and Drucker [20], a similar minimum principle was established in [21].)

The matter of uniqueness of the true solution also is of concern, and we state a pertinent result from Hill [15]. Denoting by $\Delta$ the difference between two assumed distinct solutions $u(x)$ each of which minimizes $I(v)$, there follows

$$
0=\int \Delta \tau \partial \Delta u d V \geq \int\left(\Delta \tau \mathfrak{L}^{-1} \Delta \tau+\Delta \gamma_{k} h_{k i} \Delta \gamma_{i}\right) d V
$$

Obviously, the true stress-rate $\tau$ is unique for nonnegative-definite $h_{k i}$. It turns out that $u$ and the $\gamma_{k}$ are unique as well if the critical hyperplane normals $N_{k}$ in stress space are linearly independent (at least in the discrete case, for which see [16] or [22]). This is a weaker condition than the requirement of positive-definite $h_{k i}$, which assures 
uniqueness directly from (16). (For linear independence in a general six-dimensional stress space it is sufficient that no more than six hyperplanes intersect at a critical point, or five if one defines the bounded elastic domain to lie within the deviatoric subspace.)

4. The finite-element approximation. We subdivide the body into a (large) number of tetrahedral sub-regions $q$, the finite elements. If there are planar material interfaces within the body corresponding to either different materials or abrupt changes in orientation of the same (anisotropic) material, the sub-regions $q$ are to be so chosen that no element cuts across a material interface. (The true stress-rate field $\tau$ need only satisfy $n\left(\tau_{+}-\tau_{-}\right)=0$ across an interface, where the normal $n$ points toward the arbitrarily chosen positive side.) We suppose all elements to be of equal order of magnitude in size and let $h$ denote a representative linear dimension. For the present we consider the external surface of the body to consist solely of plane faces so that $A_{D}, A_{F}$ are the same for the continuum $V$ as for the collection of finite elements whose (volume) sum is $V$.

The extension of the minimum principle to the simplest finite element class (viz. linear elements) can now be stated. Let $S^{h}$ denote the subspace in $\mathfrak{F C}^{1}$ of piecewise linear functions $v^{h}(x)$ satisfying $v^{h}=u^{I}$ on $A_{D}\left(u^{I}\right.$ the interpolate of $\left.u\right)$. We have

$$
u^{I}(x)=\sum u_{M} \varphi_{M}(x)=u(x)+O\left(h^{2}\right)
$$

within each $q$, where the summation is taken over the nodes of the element, $u_{M}$ are the true nodal displacement rates, and the $\varphi_{M}(x)$ are linear interpolation functions determined from $\varphi_{M}\left(x_{J}\right)=\hat{o}_{M J}$. Piecewise constant $\lambda_{k}{ }^{h}$ and $\zeta^{h}$ are related to $v^{h}$ through volume integrals of (11) within each element:

$$
\begin{gathered}
\int_{a^{\prime}}\left(h_{k i} \lambda_{i}{ }^{h}-N_{k} \zeta^{h}\right) d V \geq 0, \quad \lambda_{k}{ }^{h} \geq 0 \\
\int_{a^{\prime}} \lambda_{k}{ }^{h}\left(h_{k j} \lambda_{i}{ }^{h}-N_{k} \zeta^{h}\right) d V=0 \quad(\text { for each } k), \\
\zeta^{h}=\mathscr{L}\left(\partial v^{h}-N_{k} \lambda_{k}{ }^{h}\right),
\end{gathered}
$$

where the integrals are taken only over that portion $q^{\prime}$ of the element in which $\sigma(x)$ is a critical stress state. Then, the finite-element solution $u^{h}(x)$ minimizes I on the admissible class $v^{h}(x)$, where $u^{h}$ satisfies the discrete virtual work equation

$$
\int \tau^{h} \partial\left(v^{h}-u^{h}\right) d V=\left(f, v^{h}-u^{h}\right),
$$

$\tau^{h}$ being the discrete stress-rate $\mathcal{L}\left(\partial u^{h}-N_{k} \gamma_{k}{ }^{h}\right)$ of the finite element solution. The proof is merely a repetition of the steps in Sec. 3 .

In linear elasticity the corresponding minimum principle is sufficient to establish convergence of the finite-element solution. The most remarkable result is that the energy in the error over the class $v^{h}$ is minimized by $u^{h}$ (which statement is the first part of the "fundamental Theorem 1.1" in Strang and Fix [3]). Thus, for linear elasticity and the minimal finite element class (i.e. piecewise linear $v^{h}$ ),

$$
a\left(u-u^{h}, u-u^{h}\right) \leq a\left(u-v^{h}, u-v^{h}\right)=O\left(h^{2}\right)
$$


when we take $v^{h}(x)$ as the interpolate $u^{I}$ everywhere in $V$. For the elastic-plastic solid, however, it does not appear possible to establish convergence via a direct frontal attack on $I(v)$. We briefly indicate the difficulty.

From (11) and (18) and the virtual work equations (12) and (19),

$$
\begin{aligned}
a\left(u, v^{h}-u^{h}\right)-\left(\gamma, v^{h}-u^{h}\right) & =\left(f, v^{h}-u^{h}\right), \\
a\left(u^{h}, v^{h}-u^{h}\right)-\left(\gamma^{h}, v^{h}-u^{h}\right) & =\left(f, v^{h}-u^{h}\right) .
\end{aligned}
$$

(The last term in (12) is to be replaced by $\int u^{I} t(u) d A_{D}$ for both $v^{h}$ and $u^{h}$.) Thus

$$
a\left(u-u^{h}, v^{h}-u^{h}\right)=\left(\gamma-\gamma^{h}, v^{h}-u^{h}\right) .
$$

Paralleling the proof in [3] of Eq. (20) above, we obtain instead for the elastic-plastic solid:

$$
a\left(u-u^{h}, u-u^{h}\right) \leq a\left(u-v^{h}, u-v^{h}\right)+2\left(\gamma-\gamma^{h}, v^{h}-u^{h}\right) .
$$

Again, we may choose $v^{h}=u^{I}$, whence the first term on the right-hand side is $O\left(h^{2}\right)$ from (17). The problem is in the second term, where nothing can be done with either of its equivalent bilinear forms (22). Another approach is required to prove convergence, and in Secs. 5 and 6 we present the simplest one that we have found.

We close this section by briefly considering the matter of change in domain. For a body whose external surface is smoothly curved it is necessary to distinguish between $A_{D}, A_{F}, V$ for the smooth continuum and ${A_{n}}^{h},{A_{F}}^{h}, V^{h}$ for the collection of finite elements adopted here. Thus, $t^{h}$ on $A_{F}{ }^{h}$ will not necessarily equal $t$ on $A_{F}$, the domains of integration in (12) and (19) (or (21) and $(21)_{2}$ ) will differ, and relations like (22) or (23) (or even (20) in the elastic case) will not strictly hold. We have looked into the additional investigation required for this problem, but we have not carried out a rigorous analysis due to the complications associated with the present constitutive theory. In the case of linear elasticity some arguments have been given by Tong and Pian [2]. Strang and Fix [3] have suggested that if the boundary of $V^{h}$ goes systematically in and out of $V$, the error due to the change in domain will be partly self-cancelling. This is a kind of Saint-Venant principle, with the leading error term for the classical problem which they consider dependent upon the difference in volumes $V^{h}-V$. It seems likely that a similar analysis could be applied to the general elastic-plastic solid and that the errors introduced would converge to zero as $V^{h} \rightarrow V$ (with increasing number of elements). However, the convergence arguments in the following sections strictly apply only to bodies whose surfaces are piecewise plane.

5. Partitioning and convergence of the elastic solution. How do we approach the convergence proof, since a direct parallel of the steps followed in elasticity is not helpful? The answer is found in the solution scheme actually adopted for calculation of elastic-plastic solids. The boundary value problem is partitioned into two parts: a (pseudo-) elastic problem and a self-straining problem whose solution depends upon the elastic solution. Each part may be treated as a minimization problem, and it is the separate minimum principles associated with these problems that enables the convergence proof. The same basic idea was followed in [1], but there the convergence of the separate scalar functionals was related back to a convergence proof of $I\left(u^{h}\right)$ to $I(u)$. This necessitated the introduction of a fourth specific $v(x)$ in addition to $u, u^{I}, u^{h}$ (and their elastic and self-straining parts). We have avoided this extra complexity here. 
Since the convergence proof for the elastic part of the problem follows easily from the analysis in Secs. 3 and 4 (and is found in [2] and [3]), we state only essential results. Define

$$
I_{1}(v)=\frac{1}{2} a(v, v)-(f, v)
$$

over the space $\mathfrak{F}^{1}$ of continuous functions $v(x)$ satisfying $v=u$ on $A_{D}$. The statically admissible true solution $u_{\bullet}(x)$ minimizes $I_{1}$ on the admissible class $v(x)$. Further, the finiteelement solution $u_{e}{ }^{h}(x)$ minimizes both $I_{1}$ and the energy in the error over the subspace $S^{h}$ of piecewise linear functions $v^{h}(x)$ satisfying $v^{h}=u^{I}$ on $A_{D}$. Hence

$$
a\left(u_{\theta}-u_{e}^{h}, u_{\odot}-u_{e}^{h}\right) \leq a\left(u_{\theta}-v^{h}, u_{\theta}-v^{h}\right)=O\left(h^{2}\right)
$$

when we choose $v^{h}(x)$ to be the interpolate $u_{e}^{I}=u_{e}+O\left(h^{2}\right)$ of the elastic solution (with $u_{0} \equiv u$ on $A_{D}$ ). Thus the finite-element solution converges in the elastic part of the problem, and we expect (but cannot rigorously conclude) that $u_{e}{ }^{h}=u_{e}+O\left(h^{2}\right)$ on the average in $V$.

6. The self-straining solution: minimum principle and convergence proof. As the prescribed $u$ on $A_{D}, t$ on $A_{F}$, and $f$ in $V$ are included within the elastic solution, these data are identically zero in the self-straining problem. Moreover, since $\tau_{e}=\mathcal{L} \partial u_{e}$ by definition, the self-straining stress-rate field $\tau_{s}=\tau-\tau_{c}$ is related to $u_{c}=u-u_{e}$ and $\gamma_{k}$ by

$$
\tau_{s}=\mathfrak{L}\left(\partial u_{s}-N_{k} \gamma_{k}\right) .
$$

The corresponding virtual work equation is

$$
\int \tau_{s}\left(u_{s}\right) \partial w d V=0,
$$

where $w$ is any continuous vector function vanishing on $A_{D}$.

We now establish a minimum principle for the self-straining problem which is the basis of our convergence proof. Define

$$
I_{2}(w, \lambda)=\frac{1}{2} a(w, w)+\frac{1}{2} c(\lambda, \lambda)-(\lambda, w)-\left(\lambda, u_{e}\right)
$$

over the space $\mathfrak{F C}^{1}$ of continuous (vector) functions $w(x)$, identically zero on $A_{D}$, and the field of nonnegative scalar functions $\lambda(x)$, where

$$
c(\lambda, \lambda)=\int \lambda_{k}(x)\left(h_{k i}+N_{k} \mathcal{L} N_{i}\right) \lambda_{i}(x) d V .
$$

The statically admissible true solution $u_{0}(x), \gamma(x)$ satisfying the constitutive equations minimizes $I_{2}$ over the class of admissible $w(x), \lambda(x)$. We emphasize that, contrary to the case for $I(v)$, the admissible $w$ and $\lambda_{k}$ need not be related. Of course, $u_{\varepsilon}$ and $\gamma_{k}$ (and $u_{e}$ ) are related through (6)-(8) and (26). The proof follows.

We first express (28) in an equivalent but more convenient form. Defining

$$
\zeta_{.}=\mathfrak{L}\left(\partial w-N_{k} \lambda_{k}\right)
$$

we may write

$$
a(w, w)+c(\lambda, \lambda)-2(\lambda, w)=\int\left(\zeta_{\diamond} \mathcal{L}^{-1} \zeta_{\bullet}+\lambda_{k} h_{k j} \lambda_{j}\right) d V .
$$


Upon substituting into (28):

$$
I_{2}(w, \lambda)=\frac{1}{2} \int\left(\zeta_{s} \mathcal{L}^{-1} \zeta_{s}+\lambda_{k} h_{k j} \lambda_{j}\right) d V-\left(\lambda, u_{e}\right) .
$$

Secondly, we simplify $I_{2}\left(u_{s}\right)$ (so expressed because $u_{s}$ and $\gamma$ are interdependent) by using a constitutive equality from (7), (8),

$$
c(\gamma, \gamma)=(\gamma, u),
$$

and the virtual work equation (27), which may be equivalently expressed (through 26))

$$
a\left(u_{\bullet}, w\right)=(\gamma, w) .
$$

Thus, from (33) and (34) (setting $w=u_{s}$ ),

$$
I_{2}\left(u_{s}\right)=\frac{1}{2} a\left(u_{s}, u_{s}\right)-\frac{1}{2}(\gamma, u)=-\frac{1}{2}\left(\gamma, u_{e}\right),
$$

whence from (32)(with $w=u_{s}$ and $\lambda=\gamma$ )

$$
I_{2}\left(u_{s}\right)=-\frac{1}{2} \int\left(\tau_{s} \mathcal{L}^{-1} \tau_{s}+\gamma_{k} h_{k i} \gamma_{i}\right) d V .
$$

Upon subtracting (36) from (32), utilizing (27), we obtain

$$
\begin{aligned}
I_{2}(w, \lambda)-I_{2}\left(u_{s}\right)=\frac{1}{2} \int & \left\{\left(\tau_{s}-\zeta_{s}\right) \mathcal{L}^{-1}\left(\tau_{s}-\zeta_{s}\right)\right. \\
& \left.+\left(\gamma_{k}-\lambda_{k}\right) h_{k i}\left(\gamma_{i}-\lambda_{i}\right)+2 \lambda_{k}\left(h_{k i} \gamma_{i}-N_{k} \tau\right)\right\} d V \geq 0
\end{aligned}
$$

from (6) for all positive $\lambda_{k}(x)$. (Q.E.D.) This equation is reminiscent of (15) in Sec. 3, but we again stress the fundamental difference. The minimum principle on $I(v)$ depends upon the constitutive identity (33) for the admissible $v, \lambda_{k}$ (i.e. $c(\lambda, \lambda)=(\lambda, v)$ ), which requirement follows from conditions (11). The fact that the minimum principle on $I_{2}(w, \lambda)$ does not require such a relation is the key to the convergence proof.

Since we have $u_{e}{ }^{h}$ (rather than $u_{e}$ ) from the elastic finite-element solution, the scalar functional actually minimized in computations of the self-straining problem is

$$
I_{2}{ }^{*}(w, \lambda)=\frac{1}{2} a(w, w)+\frac{1}{2} c(\lambda, \lambda)-(\lambda, w)-\left(\lambda, u_{e}{ }^{h}\right),
$$

where

$$
I_{2}{ }^{*}(w, \lambda)=I_{2}(w, \lambda)+\left(\lambda, u_{e}-u_{e}{ }^{h}\right) .
$$

Paralleling the principle above, the following theorem on $I_{2}{ }^{*}$ may be stated. The finite element solution $u_{s}{ }^{h}(x), \gamma^{h}(x)$ minimizes $I_{2}{ }^{*}$ over the subspace $S^{h}$ of piecewise linear functions $w^{h}(x)$, identically zero on $A_{D}$, and the field of nonnegative scalar functions $\lambda(x)$. Eqs. (30) through (36) follow as before. Typically,

$$
\begin{aligned}
I_{2}{ }^{*}\left(w^{h}, \lambda\right) & =\frac{1}{2} \int\left(\zeta_{s}{ }^{h} \mathcal{L}^{-1} \zeta_{s}{ }^{h}+\lambda_{k} h_{k j} \lambda_{j}\right) d V-\left(\lambda, u_{e}{ }^{h}\right), \\
c\left(\gamma^{h}, \gamma^{h}\right) & =\left(\gamma^{h}, u^{h}\right), \quad a\left(u_{s}{ }^{h}, w^{h}\right)=\left(\gamma^{h}, w^{h}\right), \\
I_{2}{ }^{*}\left(u_{s}{ }^{h}\right) & =-\frac{1}{2}\left(\gamma^{h}, u_{e}{ }^{h}\right)=-\frac{1}{2} \int\left(\tau_{s}{ }^{h} \mathcal{L}^{-1} \tau_{s}{ }^{h}+\gamma_{k}{ }^{h} h_{k} \gamma_{i}{ }^{h}\right) d V,
\end{aligned}
$$


(note that $\zeta_{s}{ }^{h}$ is not necessarily piecewise constant although $\tau_{s}{ }^{h}$ is) and the proof of the theorem is completed by

$$
\begin{aligned}
I_{2}{ }^{*}\left(w^{h}, \lambda\right)-I_{2}{ }^{*}\left(u_{s}{ }^{h}\right) & =\frac{1}{2} \int\left\{\left(\tau_{s}{ }^{h}-\zeta_{s}{ }^{h}\right) \mathfrak{L}^{-1}\left(\tau_{s}{ }^{h}-\zeta_{s}{ }^{h}\right)\right. \\
& \left.+\left(\gamma_{k}{ }^{h}-\lambda_{k}\right) h_{k j}\left(\gamma_{i}{ }^{h}-\lambda_{j}\right)+2 \lambda_{k}\left(h_{k i} \gamma_{i}{ }^{h}-N_{k} \tau^{h}\right)\right\} d V \geq 0 .
\end{aligned}
$$

We now have all the results necessary to establish convergence. Denoting the nonnegative form on the right-hand side of (37) by $\left(w, \lambda ; u_{s}, \gamma\right)$, there follows for the class $w^{h}$

$$
I_{2}\left(w^{h}, \lambda\right)-I_{2}\left(u_{s}\right)=\left(w^{h}, \lambda ; u_{s}, \gamma\right) \geq 0 .
$$

Further, from (39) and (43),

$$
I_{2}\left(w^{h}, \lambda\right)+\left(\lambda-\gamma, u_{e}-u_{e}^{h}\right) \geq I_{2}\left(u_{s}^{h}\right) .
$$

But (44) also holds for the finite-element solution $u_{s}{ }^{h}, \gamma^{h}$, whence $I_{2}\left(u_{s}{ }^{h}\right) \geq I_{2}\left(u_{s}\right)$ and we obtain the continued inequality

$$
I_{2}\left(w^{h}, \lambda\right)-I_{2}\left(u_{s}\right)+\left(\lambda-\gamma^{h}, u_{e}-u_{e}{ }^{h}\right) \geq I_{2}\left(u_{s}{ }^{h}\right)-I_{2}\left(u_{s}\right) \geq 0,
$$

or, from (44),

$$
\left(w^{h}, \lambda ; u_{s}, \gamma\right)+\left(\lambda-\gamma^{h}, u_{e}-u_{e}^{h}\right) \geq\left(u_{s}^{h}, \gamma^{h} ; u_{s}, \gamma\right) \geq 0 .
$$

Since $w^{h}, \lambda$ are unrelated, we may choose $w^{h}=u_{s}{ }^{I}$, the $O\left(h^{2}\right)$ interpolate, and $\lambda_{k}=\gamma_{k}$, the actual values. Then, from (7) and (31), (47) reduces to

$$
\frac{1}{2} a\left(u_{s}-u_{s}^{I}, u_{s}-u_{s}^{I}\right)+\left(\gamma-\gamma^{h}, u_{e}-u_{e}^{h}\right) \geq\left(u_{s}^{h}, \gamma^{h} ; u_{s}, \gamma\right) \geq 0 .
$$

The first term is $O\left(h^{2}\right)$. Thus we may write

$$
\begin{aligned}
\frac{1}{2} \int\left(\tau_{s}-\tau_{s}{ }^{h}\right) \mathcal{L}^{-1}\left(\tau_{s}\right. & \left.-\tau_{s}{ }^{h}\right) d V+\frac{1}{2} \int\left(\gamma_{k}-\gamma_{k}{ }^{h}\right) h_{k i}\left(\gamma_{i}-\gamma_{i}{ }^{h}\right) d V \\
& +\int \gamma_{k}{ }^{h}\left(h_{k} \gamma_{i}-N_{k} \tau\right) d V \leq\left(\gamma-\gamma^{h}, u_{e}-u_{e}{ }^{h}\right)+O\left(h^{2}\right) .
\end{aligned}
$$

This is the principal new and substantive result of our analysis. Recalling that

$$
a\left(u_{e}-u_{e}{ }^{h}, u_{e}-u_{e}{ }^{h}\right) \leq O\left(h^{2}\right)
$$

from (25), $u_{e}^{h} \rightarrow u_{e}$ and the right-hand side converges to zero. Thus, the nonnegative integrals on the left-hand side must individually converge to zero. The immediate conclusions are that $\tau_{s}{ }^{h}$ converges to $\tau_{s}$, consequently $\tau^{h} \rightarrow \tau$, and ${\gamma_{k}}^{h}$ converges to a positive value wherever $\gamma_{k}$ is positive (i.e. the same hyperplanes are activated). This latter condition suffices to make the third integral converge to zero since $h_{k i} \gamma_{i}=N_{k} \tau$ if $\gamma_{k}>0$. In addition,

$$
\left(\gamma_{k}-\gamma_{k}{ }^{h}\right) h_{k i}\left(\gamma_{i}-\gamma_{i}{ }^{h}\right) \rightarrow 0
$$

and, from $\tau_{s}{ }^{h} \rightarrow \tau_{s}$,

$$
\partial\left(u_{s}-u_{s}^{h}\right) \rightarrow N_{k}\left(\gamma_{k}-\gamma_{k}^{h}\right) .
$$

For positive-definite $h_{k i}$ the $\gamma_{k}{ }^{h}$ unquestionably converge to $\gamma_{k}$, and $u_{s}{ }^{k}$ converges to $u_{s}$. For positive-sem $i$ definite $h_{k i}$ there conceivably may be nonvanishing $\gamma_{k}-\gamma_{k}{ }^{h}$ which satisfy both equations in the limit, but this seems to us unlikely. (In the case of Taylor hardening (50) reduces to $\sum\left(\gamma_{k}-\gamma_{k}{ }^{k}\right) \rightarrow 0$, requiring at least that errors in the $\gamma_{k}$ algebraically sum to zero at each critical stress point. The analysis in [1] further indicates 
that the individual $\gamma_{k}{ }^{h}$ converge. The other specific hardening rules which have been proposed are positive-definite.)

Lastly, we observe that the following orders of error would be consistent with (49): $\tau_{s}{ }^{h}=\tau_{s}+O(h), \gamma_{k}{ }^{h}=\gamma_{k}+O(h), u_{s}{ }^{h}=u_{s}+O\left(h^{2}\right)$. Of course these, as well as $u_{e}{ }^{h}=$

$u_{e}+O\left(h^{2}\right)$ (hence $\tau_{e}{ }^{h}=\tau_{e}+O(h)$ ), have not been proved, but we believe they should be expected.

Acknowledgment. We thank the referee for valuable suggestions and comments on the first version of this work.

\section{REFERENCES}

[1] K. S. Havner, On convergence of a discrete aggregate model in polycrystalline plasticity, Int. J. Solids Structures 7, 1269-1275 (1971)

[2] P. Tong and T. H. H. Pian, The convergence of finite element method in solving linear elastic problems, Int. J. Solids Structures 3, 865-879 (1967)

[3] G. Strang and G. J. Fix, An analysis of the finite element method, Prentice-Hall, New Jersey (1973)

[4] G. I. Taylor, The distortion of crystals of aluminum under compression, Part II, Proc. R. Soc. Lond. A116, 16-38 (1927)

[5] G. I. Taylor, Plastic strain in metals, J. Inst. Metals 62, 307-324 (1938)

[6] J. F. W. Bishop and R. Hill, A theoretical derivation of the plastic properties of a polycrystalline face-centered metal, Phil. Mag. (7) 42, 1298-1307 (1951)

[7] T. H. Lin, Physical theory of plasticity, in Advances in applied mechanics, Vol. 11 (edited by C.-S. Yih), Academic Press, New York, 255-311 (1971)

[8] J. W. Hutchinson, Plastic stress-strain relations of F.C.C. polycrystalline metals hardening according to T'aylor's rule, plastic deformation of B.C.C. polycrystals, J. Mech. Phys. Solids 12, 11-33 (1964)

[9] J. W. Hutchinson, Elastic-plastic behaviour of polycrystalline metals and composites, Proc. R. Soc. Lond. A319, 247-272 (1970)

[10] K. S. Havner and R. Varadarajan, A quantitative study of a crystalline aggregate model, Int. J. Solids Structures 9, 379-394 (1973)

[11] K. S. Havner, C. Singh and R. Varadarajan, Plastic deformation and latent strain energy in a polycrystalline aluminum model, Int. J. Solids Structures 10, 853-862 (1974)

[12] B. Budiansky and T. T. Wu, Theoretical prediction of plastic strains of polycrystals, Proc. 4th U.S. Nat. Cong. Appl. Mech., ASME, 1175-1185 (1962)

[13] W. T. Koiter, Stress-strain relations, uniqueness and variational theorems for elastic-plastic materials with a singular yield surface, Quart. Appl. Math. 11, 350-354 (1953)

[14] J. Mandel, Generalization de la théorie de plasticité de W. T. Koiter, Int. J. Solids Structures 1, 27:3-295 (1965)

[15] R. Hill, Generalized constitutive relations for incremental deformation of metal crystals by multislip, J. Mech. Phys. Solids 14, 95-102 (1966)

[16] G. Maier, A quadratic programming approach for certain nonlinear structural problems, Meccanica 3, 121-130 (1968)

[17] G. Maier, "Linear" flow-laws of elastoplasticity: A unified general approach, Lincei-Rend. Sci. Fis. Mat. e Nat. (8) 47, 267-276 (1969)

[18] O. Del)onato and A. Franchi, A modified gradient method for finite element elastoplastic analysis by quadratic programming, Comp. Meth. Appl. Mech. Engng. 2, 107-131 (1973)

[19] W. Prager, Recent developments in the mathematical theory of plasticity, J. Appl. Phys. 20, 235-241 (1949)

[20] D. C. Drucker, Some implications of work hardening and ideal plasticity, Quart. Appl. Math. 7, 411-418 (1950)

[21] 1). C. Drucker, Variational principles in the mathematical theory of plasticity, Proc. Symposia Appl. Math. 8, MeGraw-Hill, New York, 7-22 (1958)

[22] K.S. Havner, A discrete model for the prediction of subsequent yield surfaces in polycrystalline plasticity, Int. J. Solids Structures 7, 719-730 (1971) 